\title{
BMJ Open Epidemiology, clinical characteristics and treatment of critically ill patients with COVID-19): a protocol for a living systematic review
}

\author{
Lazar Milovanovic (D) , ${ }^{1}$ Erin Hessey, ${ }^{2}$ Meghan Sebastianski (D) , ${ }^{3}$ \\ Diana Keto-Lambert, ${ }^{3,4}$ Ben Vandermeer, ${ }^{3,4}$ Sean M Bagshaw, ${ }^{1}$ Oleksa Rewa ${ }^{1}$
}

To cite: Milovanovic L, Hessey E, Sebastianski M, et al. Epidemiology, clinical characteristics and treatment of critically ill patients with COVID-19): a protocol for a living systematic review. BMJ Open 2021;11:e042008. doi:10.1136/ bmjopen-2020-042008

- Prepublication history and additional material for this paper is available online. To view these files, please visit the journal online (http://dx.doi.org/10. 1136/bmjopen-2020-042008).

Received 23 June 2020 Revised 17 November 2020 Accepted 23 November 2020

Check for updates

(C) Author(s) (or their employer(s)) 2021. Re-use permitted under CC BY-NC. No commercial re-use. See rights and permissions. Published by BMJ.

${ }^{1}$ Department of Critical Care Medicine, Faculty of Medicine and Dentistry, University of Alberta, Edmonton, Alberta, Canada

${ }^{2}$ Faculty of Medicine and Dentistry, University of Alberta, Edmonton, Alberta, Canada ${ }^{3}$ Alberta Strategy for PatientOriented Research (SPOR) Knowledge Translation Platform, University of Alberta, Edmonton, Alberta, Canada

${ }^{4}$ Alberta Research Center for Health Evidence (ARCHE), University of Alberta, Edmonton, Alberta, Canada

Correspondence to

Dr Oleksa Rewa;

rewa@ualberta.ca

\section{ABSTRACT}

Introduction In December 2019, the first cases of COVID-19 associated with SARS-CoV-2 viral infection were described in Wuhan, Hubei Province, China. Since then, it has spread rapidly affecting 188 countries and was declared a pandemic by the WHO on 11 March 2020. Preliminary reports suggest up to $30 \%$ of patients require intensive care unit (ICU) admission and case fatality rate estimate is $2.3 \%-7.2 \%$. The primary reason for ICU admission is hypoxaemic respiratory failure, while factors associated with ICU admission include increased age, presence of comorbidities and cytokine storm. Case series and retrospective trials initially assessed proposed treatments with randomised controlled trials now reporting early outcomes. We conduct a systematic review and meta-analysis to identify epidemiological factors, treatments and complications that predict mortality among critically ill patients with COVID-19.

Methods and analysis Our comprehensive search strategy was developed in consultation with a research librarian. We will search electronic databases: Ovid Medline, Ovid Embase, Ovid Cumulative Index to Nursing and Allied Health Literature (CINAHL) and Wiley Cochrane Library. The search strategy combines concepts from COVID-19, validated COVID-19 search filters and geographical locations of large outbreaks. Citation screening, selection, quality assessment and data abstraction will be performed in duplicate. Clinically homogenous epidemiological characteristics, interventions and complications will be pooled in statistical metaanalysis. Within the framework of a living systematic review, the search and data analysis will be updated every 6 months.

Ethics and dissemination Our systematic review will synthesise literature on risk factors and interventions associated with mortality in critically ill patients with COVID-19. Results will be presented at national and international conferences and submitted for peer-reviewed publication. The pooled analysis can provide guidance to inform clinical guidelines for care of critically ill patients with COVID-19. Iterative updates will be made public through open access. Research ethics approval is not required.

PROSPERO registration number CRD42020176672.
Strengths and imitations of this study

- This systematic review protocol follows the Preferred Reporting Items for Systematic Review and MetaAnalysis Protocols guidelines conducting a rigorous risk of bias assessment.

- This systematic review synthesises patient data from a variety of time points in the pandemic (early retrospective data, emerging prospective cohort studies and randomised controlled trials) and from a large number of centres worldwide, providing clarity for a topic with a large volume of noise in the literature.

- Non-English electronic databases will not be searched, potentially leading to language bias in this study.

- There is the potential for inclusion of low and inconsistent quality data from small, limited early studies due to the rapid nature of the spread and the high acuity of the COVID-19 pandemic.

\section{INTRODUCTION}

In December 2019, the first cases of COVID-19 associated with SARS-CoV-2 viral infection were described in Wuhan, Hubei Province, China. ${ }^{1}$ Initially associated with an outbreak of atypical pneumonia from the Hunan Seafood Wholesale Market in Wuhan city of China, direct human to human spread via aerosolisation was confirmed shortly after the initial outbreak. ${ }^{2-4}$ Since then, the disease has spread rapidly worldwide affecting 188 countries, and was declared a pandemic by the WHO on 11 March $2020 .^{5}$ It is associated with high rates of hospitalisation, with estimates suggesting $5 \%-30 \%$ of patients are admitted to an intensive care unit (ICU). ${ }^{6} 7$ As of 25 October 2020, the WHO reports that over 43 million people have been infected and over 1.1 million have died of COVID-19.

The rapid spread of COVID-19 cases has created significant burden for healthcare systems across the world. ${ }^{9-11}$ Rapid 
progression of early cases in Wuhan, China resulted in insufficient resources for critically ill patients with COVID19 , delaying time to initiation of mechanical ventilation. ${ }^{12}$ Multiple countries have developed allocation protocols for the distribution of limited intensive care resources. ${ }^{13}$ As the number of critically ill patients with COVID-19 increases, the number and organisation of available ICU beds become essential to effective patient care ${ }^{14}$ Globally, the disease has led to closure of borders and implementation of strict social and physical distancing. ${ }^{15-17}$ Economically, COVID-19 has had a major impact on several aspects of the worldwide economy including agriculture, petroleum and oil, manufacturing and finance. ${ }^{18}$

Within the reported critically ill COVID-19 data, there is substantial variation in population characteristics, duration of follow-up and interventions assessed across studies due to differences in design, case-mix and data quality. ${ }^{19}{ }^{20}$ Preliminary epidemiological data, reports and retrospective observational data suggest a COVID-19 case fatality rate ranging from $2.3 \%$ to $7.2 \% .^{1321} 22$ The leading cause of mortality is acute respiratory distress syndrome (ARDS). ${ }^{23}$ Factors associated with critical illness and admission to ICU include increased age, presence of comorbidities, lymphopenia and elevated Lactate Dehydrogenase (LDH), D-dimer and ferritin concerning for cytokine storm. ${ }^{24-28}$

Multiple treatment modalities have been proposed including hydroxychloroquine, dexamethasone, azithromycin, multiple antiviral and retroviral agents as well as blood purification techniques. ${ }^{29-33}$ As of the publication of this protocol, there are over 1300 prospective observational and randomised controlled trials registered in clinical trials databases targeting critically ill patients with COVID-19. ${ }^{34-37}$ As prospective and randomised data becomes available, synthesis of intervention outcomes across different populations will be essential for the development of high-quality evidence for treatment and care of these patients.

We previously conducted a rapid review of the literature on critically ill patients with COVID-19 approximately 4 months after the first cases were reported. ${ }^{38}$ Limited to small case series, retrospective observational studies and small prospective cohort studies, our review found that increased mortality is associated with acute kidney injury, acute cardiac injury and the presence of shock. These data serve as an initial perspective on factors affecting mortality in critically ill patients with COVID-19 and the treatment strategies being used. ${ }^{31} 39$

We will conduct a systematic review and meta-analysis to identify risk factors, epidemiological characteristics, treatments and complications that predict mortality among patients with COVID-19 admitted to ICUs (hereby defined as critically ill patients with COVID-19). This will address ongoing research into therapies such as antiviral agents and glucocorticoids while allowing time for data synthesis from the first wave to be completed. The ongoing development and publication of prospective and randomised data will serve as a robust dataset from which synthesis and meta-analysis will provide stronger recommendations for ongoing care and management of critically ill patients with COVID-19.

\section{METHODS}

\section{Patient and public involvement}

No patient or public will be involved in this systematic review and meta-analysis.

\section{Study design}

A systematic review will be performed using guidelines from The Cochrane Collaboration and Center for reviews, and disseminated and described according to Preferred Reporting Items for Systematic Reviews and Meta-Analyses Protocol guidelines https://systematicre viewsjournal.biomedcentral.com/articles/10.1186/ 2046-4053-4-1.

\section{Study registration}

This systematic review is registered with PROSPERO (CRD42020176672) (2 April 2020).

\section{Data source and search methods}

The search strategy will be developed and performed in consultation with a research librarian and peer reviewed with a second research librarian. We will search electronic databases: Ovid Medline (1946-), Ovid Embase (1988-), CINAHL (1937-) and Wiley Cochrane Library (inception-). Additional search sources will include the trial registry platforms (ie, ClinicalTrials.gov), Google Scholar and preprint literature databases (ResearchSquare, Preprints.org, MedRxiv, bioRxiv and EPPI-Centre) as data on COVID-19 is rapidly being published. The table of contents of journals frequently publishing COVID-19 data, as well as reference lists will be reviewed. The search strategy combines concepts from COVID-19 (ie, SARS-CoV-2, COVID-19, coronavirus, 2019nCoV), geographic locations of large outbreaks (Wuhan, Hubei, Huanan, Italy, Washington, Seattle, London, Sao Paolo and New York) and critical care (ie, ICU, intensive care, critical care, mechanical ventilator, ARDS, extracorporeal membrane oxygenation, ECMO). A validated search filter for COVID-19 from the Center for Disease Control and Prevention will be incorporated (https://www.cdc. gov/library/researchguides/2019novelcoronavirus/ pubmedsearchalert.html). An earlier search strategy was validated across a subset of relevant articles and used for a COVID-19 rapid review performed by this group. ${ }^{38}$ It will be used as the basis for our search strategy in this review. See online supplemental table 1 for the Ovid Medline search strategy.

\section{Study selection}

Eligible articles will be identified through two phases. In the first phase, two authors will independently review the titles and abstracts of all retrieved articles and documents using Covidence systematic review software (Veritas Health Innovation, Melbourne, Australia) for potential 
inclusion. Disagreements will be resolved through discussion or adjudication by a third author. Full texts of selected articles will be reviewed by the same two authors independently and reviewed for eligibility using standard, predefined criteria. Disagreements will be resolved through discussion with a third author.

\section{Eligibility criteria}

Studies will be included if they present primary clinical data including outcomes of critically ill patients with laboratory-confirmed COVID-19 undergoing intervention or receiving treatment for COVID-19. Prospective observational and randomised control trials will be included. Paediatric studies and patients under the age of 18 will be excluded. We will exclude systematic reviews, secondary analyses of previously published data, narrative reviews, editorials and case reports. Descriptive studies without outcomes will be excluded.

\section{Outcome measures}

The primary outcome will be the case fatality rate among critically ill patients with COVID-19. This will be further broken down into ICU-specific mortality, in-hospital mortality, 28-day mortality and 90-day mortality. Where available, deaths per 1000 ICU admissions will be collected. Mortality outcomes will be stratified by Acute Physiologic Assessment and Chronic Health Evaluation II score, the Sequential Organ Failure Assessment score and critical care interventions (high-flow nasal oxygen, non-invasive mechanical ventilation, invasive mechanical ventilation, ECMO and renal replacement therapy). Secondary outcomes include duration of mechanical ventilation, duration of stay in the ICU, discharge destination and functional status (characterised by patientreported function, that is, health-related quality of life) at time of discharge. Primary and secondary outcomes will be assessed as a function of care processes including advanced ventilator modalities (eg, airway pressure release ventilation), proning, use of muscle relaxants and inhaled pulmonary vasodilators as well as COVID-19 specific treatments including antibiotics, antivirals and immunomodulatory agents (ie, anti-interleukin 6 (antiIL6) agents, glucocorticoids).

\section{Data extraction}

A structured data extraction tool will be developed and piloted to extract study-specific data. Data will be extracted independently by two authors. Data abstracted will include publication details, study design, centre/ treatment hospital, study period, patient epidemiological characteristics, clinical presentation at time of ICU admission, COVID-19 specific treatments, ICU interventions, complications occurring and outcomes. Disagreements will be resolved through discussion or adjudication by a third author. The authors of the retrieved studies and/ or documents will be contacted for further information if necessary. If two studies use patients from the same time period and centre/treatment hospital, the authors will be contacted to assess for potential patient overlap.

\section{Risk-of-bias assessment}

Study methodological quality will be independently rated by two authors using the Newcastle Ottawa Scale for observational studies and the Cochrane Collaboration's tool for randomised controlled trials. ${ }^{4041}$ Preprint studies will be excluded until publication in a peer-reviewed journal. The strength of outcome measures derived from each study will be evaluated using the Grading of Recommendations, Assessment, Development and Evaluations system (clinicalevidence.bmj.com/x/set/static/ebm/ learn/665072.html) and the Meta-analysis Of Observational Studies in Epidemiology checklist for meta-analysis and systematic reviews of observational studies. ${ }^{42}$

\section{Data analysis}

Studies will be evaluated for clinical heterogeneity and, where possible, be pooled in statistical meta-analysis. Observational data will be pooled together and RCT data will be pooled together. Two by two tables will be constructed from treatments, epidemiological data and complications with outcomes reported in each study. If not reported, authors will be contacted for the data and this will be used calculate missing statistical parameters from reported values. If adjusted and unadjusted results are presented in the studies, unadjusted data will preferentially be used for meta-analysis. Where results are reported as risk ratios and it is not possible to construct $2 \times 2$ tables from the available data, they will not be included in the statistical meta-analysis. If sufficient data are available, subgroup analysis including separating data by continent, age (patients $<65$ and $>65$ ), those undergoing mechanical ventilation and ECMO will be completed. For epidemiological factors, biochemical parameters (ie, D-Dimer, C reactive protein, B-natriuretic peptide, IL-6 level, ferritin, albumin), treatments or complications with four or more sufficiently clinically homogenous studies reporting, a meta-analysis will be conducted.

We will use a random effects model to pool effect sizes for each exposure as it associates with the primary outcome. Dichotomous outcomes will be reported as pooled risk ratios and 95\% CIs based on the DerSimonianLaird random-effect model. ${ }^{43}$ Continuous outcomes will be reported using calculated weighted mean differences with their $95 \%$ CIs. Using the $\mathrm{I}^{2}$ statistic and $\chi^{2}$ test, we will assess heterogeneity among studies. The thresholds for interpretation of $\mathrm{I}^{2}$ will be in accordance with the definitions presented in the Cochrane Handbook for Systematic Reviews of Interventions. ${ }^{44}$

\section{ETHICS AND DISSEMINATION}

Our systematic review will synthesise literature on risk factors and treatments associated with increased mortality in critically ill patients with COVID-19. Results will be presented at national and international conferences in 
the form of poster or oral presentations. The final manuscript will be published in a peer-reviewed journal. Iterative updates for this living systematic review will be conducted every 6 months and will be made public through digital channels and open access. The pooled analysis from this study can provide guidance to update clinical guidelines for the management of critically ill patients with COVID19. Research ethics approval is not required.

\section{DISCUSSION}

Since its initial outbreak in Wuhan, China in December 2019, COVID-19 has rapidly spread to over 188 countries and has been declared a global pandemic by the WHO. ${ }^{15}$ A significant proportion of cases require hospitalisation and between $5 \%$ and $30 \%$ of cases require admission to an ICU. ${ }^{6}$ In addition to the effect on individuals, COVID-19 has had a major impact on the worldwide economy, national borders and foreign policy. ${ }^{15} 1618$

The initial data from affected patients is limited to case reports, small series and retrospective analyses at substantial risk of bias. ${ }^{7}$ Proposed treatment targets were initially based on extrapolated data from SARS and Middle East Respiratory Syndrome, as well as observational data from small patient data sets. ${ }^{45} 46$ Observational data and early systematic reviews using non-randomised data suggest that male sex, increasing age and presence of comorbidities are epidemiological risk factors for mortality in patients admitted to ICU.$^{38} 478$ Intensive care complications associated with increased mortality include acute kidney injury, ARDS, acute cardiac injury and shock. ${ }^{38} 47$

There are several potential COVID-19 treatments currently being evaluated in a randomised fashion including anti-IL6 therapy (tociluzmab), antiviral therapy (remdesivir) and ICU specific therapies such as awake proning, prone ventilation and ECMO. ${ }^{39} 4950$ Synthesis of these interventions and outcomes across different populations and regions are essential for development of robust and up to date evidence-based guidelines for treatment of these patients. The presence of large, multinational platform trials including a randomised, embedded, multifactorial, adaptive platform trial for community-acquired pneumonia, SOLIDARITY (an international randomised controlled trial to evaluate non-licensed COVID-19 treatments in addition to standard of care), and COVID-19 ring-based prevention trial with combination lopinavir ritonavir will allow for comparisons of both COVID-19 specific and intensive care-based treatments for this disease ${ }^{51-53}$ This systematic review will use these platform trials to synthesise data and evaluate outcomes across multiple patient populations and geographical locations.

\section{Limitations and strengths}

There are several limitations to this systematic review and the data available. The first major limitation is the presence of early, small clinical trials developed during a rapidly changing clinical situation and evolving observational data. Many of these do not include recently observed complications including venous thromboembolic disease and Kawasaki-like syndrome in children. ${ }^{545}$ The development of clinical trials and prospective observational datasets has been limited by the rapidity of onset of COVID-19 and the resultant strain on the healthcare system, and the design of these studies has been tailored to maximise broad utilisation of potentially effective treatments. As of the publication of this article, over 31 million cases of COVID-19 are closed.$^{56}$ The large number of resolved cases for which limited data were collected will significantly limit the generalisability of future data collection and will increase heterogeneity of the dataset.

Strengths of this review are the ability to aggregate studies from a large number of centres with diverse patient populations during a finite time interval with similar critical care resources. The presence of multiple areas (Wuhan, Italy, New York, Sao Paolo, UK) with large clusters of cases will allow for increased generalisability of pooled data relative to individual population assessments.

Contributors This systematic review was conceived and designed by OGR, MS and SMB. This protocol was initially drafted by LM and EH. Literature search strategy was developed by MS and DK-L. Statistical analysis was provided by BV. Subsequent drafts were commented on and revisions made by the authors. All authors have approved submission.

Funding This research received in-kind from the Alberta SPOR SUPPORT Unit and the University of Alberta (grant/award number not applicable). SMB is supported by a Canada Research Chair in Critical Care Nephrology.

Competing interests None declared.

Patient consent for publication Not required.

Provenance and peer review Not commissioned; externally peer reviewed.

Supplemental material This content has been supplied by the author(s). It has not been vetted by BMJ Publishing Group Limited (BMJ) and may not have been peer-reviewed. Any opinions or recommendations discussed are solely those of the author(s) and are not endorsed by BMJ. BMJ disclaims all liability and responsibility arising from any reliance placed on the content. Where the content includes any translated material, BMJ does not warrant the accuracy and reliability of the translations (including but not limited to local regulations, clinical guidelines, terminology, drug names and drug dosages), and is not responsible for any error and/or omissions arising from translation and adaptation or otherwise.

Open access This is an open access article distributed in accordance with the Creative Commons Attribution Non Commercial (CC BY-NC 4.0) license, which permits others to distribute, remix, adapt, build upon this work non-commercially, and license their derivative works on different terms, provided the original work is properly cited, appropriate credit is given, any changes made indicated, and the use is non-commercial. See: http://creativecommons.org/licenses/by-nc/4.0/.

\section{ORCID iDs}

Lazar Milovanovic http://orcid.org/0000-0001-9294-5199

Meghan Sebastianski http://orcid.org/0000-0002-5206-8731

\section{REFERENCES}

1 WHO. Pneumonia of unknown cause - China World Health organization: World Health organization, 2020. Available: https:// www.who.int/csr/don/05-january-2020-pneumonia-of-unkowncause-china/en/ [Accessed 24 May 2020].

2 Wang C, Horby PW, Hayden FG, et al. A novel coronavirus outbreak of global health concern. The Lancet 2020;395:470-3.

3 Phan LT, Nguyen TV, Luong QC, et al. Importation and human-tohuman transmission of a novel coronavirus in Vietnam. $N$ Engl J Med 2020;382:872-4.

4 Riou J, Althaus CL. Pattern of early human-to-human transmission of Wuhan 2019 novel coronavirus (2019-nCoV), December 2019 to January 2020. Eurosurveillance 2020;25:2000058. 
5 WHO. WHO Timeline - COVID-19, 2020. Available: https://www.who. int/news-room/detail/27-04-2020-who-timeline-covid-19 [Accessed 6 Jun 2020].

6 Wu Z, McGoogan JM. Characteristics of and Important Lessons From the Coronavirus Disease 2019 (COVID-19) Outbreak in China: Summary of a Report of 72314 Cases From the Chinese Center for Disease Control and Prevention. JAMA 2020;323:1239-42.

7 Huang C, Wang Y, Li X, et al. Clinical features of patients infected with 2019 novel coronavirus in Wuhan, China. The Lancet 2020;395:497-506.

8 WHO. Coronarvirus disease 2019 (COVID-19) Weekly epidemiological, 2020. Available: https://www.who.int/docs/defaultsource/coronaviruse/situation-reports/weekly-epi-update-11.pdf [Accessed 27 Oct 2020]

9 Grasselli G, Zangrillo A, Zanella A, et al. Baseline characteristics and outcomes of 1591 patients infected with SARS-CoV-2 admitted to ICUs of the Lombardy region, Italy. JAMA 2020;323:1574-81.

10 Grasselli G, Pesenti A, Cecconi M. Critical care utilization for the COVID-19 outbreak in Lombardy, Italy: early experience and forecast during an emergency response. JAMA 2020;323:1545-6.

11 Moghadas SM, Shoukat A, Fitzpatrick MC, et al. Projecting Hospital utilization during the COVID-19 outbreaks in the United States. Proc Natl Acad Sci U S A 2020;117:9122-6.

12 Du R-H, Liu L-M, Yin W, et al. Hospitalization and critical care of 109 decedents with COVID-19 pneumonia in Wuhan, China. Ann Am Thorac Soc 2020;17:839-46.

13 Emanuel EJ, Persad G, Upshur R, et al. Fair allocation of scarce medical resources in the time of Covid-19. N Engl J Med Overseas Ed 2020;382:2049-55.

14 Palamim CVC, Marson FAL. COVID-19 - The Availability of ICU Beds in Brazil during the Onset of Pandemic. Ann Glob Health 2020;86:100

15 Wells CR, Sah P, Moghadas SM, et al. Impact of international travel and border control measures on the global spread of the novel 2019 coronavirus outbreak. Proc Natl Acad Sci U S A 2020;117:7504-9.

16 Briscese G, Lacetera N, Macis M, et al. Compliance with covid-19 social-distancing measures in Italy: the role of expectations and duration. National Bureau of Economic Research, 2020.

17 Lewnard JA, Lo NC. Scientific and ethical basis for social-distancing interventions against COVID-19. Lancet Infect Dis 2020;20:631-3.

18 Nicola M, Alsafi Z, Sohrabi C, et al. The socio-economic implications of the coronavirus pandemic (COVID-19): a review. Int J Surg 2020;78:185-93.

19 Giordano G, Blanchini F, Bruno R, et al. Modelling the COVID-19 epidemic and implementation of population-wide interventions in Italy. Nat Med 2020;26:855-60.

20 Wadhera RK, Wadhera P, Gaba P, et al. Variation in COVID-19 hospitalizations and deaths across New York City boroughs. JAMA 2020;323:2192

21 Guan W-J, Ni Z-Y, Hu Y, et al. Clinical characteristics of coronavirus disease 2019 in China. N Engl J Med 2020;382:1708-20.

22 Onder G, Rezza G, Brusaferro S. Case-Fatality rate and characteristics of patients dying in relation to COVID-19 in Italy. JAMA 2020;323:1775-6.

23 Yang X, Yu Y, Xu J, et al. Clinical course and outcomes of critically ill patients with SARS-CoV-2 pneumonia in Wuhan, China: a singlecentered, retrospective, observational study. Lancet Respir Med 2020;8:475-81.

24 Ruan Q, Yang K, Wang W, et al. Clinical predictors of mortality due to COVID-19 based on an analysis of data of 150 patients from Wuhan, China. Intensive Care Med 2020:1-3.

25 Zhou F, Yu T, Du R, et al. Clinical course and risk factors for mortality of adult inpatients with COVID-19 in Wuhan, China: a retrospective cohort study. Lancet 2020;395:1054-62.

26 Wu C, Chen X, Cai Y, et al. Risk factors associated with acute respiratory distress syndrome and death in patients with coronavirus disease 2019 pneumonia in Wuhan, China. JAMA Intern Med 2020;180:934.

27 Chen G, Wu D, Guo W, et al. Clinical and immunological features of severe and moderate coronavirus disease 2019. J Clin Invest 2020;130:2620-9.

28 Zhang JJY, Lee KS, Ang LW, et al. Risk factors for severe disease and efficacy of treatment in patients infected with COVID-19: a systematic review, meta-analysis, and meta-regression analysis. Clin Infect Dis 2020;71:2199-206.

29 Liu J, Cao R, Xu M, et al. Hydroxychloroquine, a less toxic derivative of chloroquine, is effective in inhibiting SARS-CoV-2 infection in vitro. Cell Discov 2020;6.

30 Wang $\mathrm{M}$, Cao R, Zhang L, et al. Remdesivir and chloroquine effectively inhibit the recently emerged novel coronavirus (2019nCoV) in vitro. Cell Res 2020;30:269-71.
31 Gautret P, Lagier J-C, Parola P, et al. Hydroxychloroquine and azithromycin as a treatment of COVID-19: results of an openlabel non-randomized clinical trial. Int J Antimicrob Agents 2020;56:105949

32 Ma J, Xia P, Zhou Y, et al. Potential effect of blood purification therapy in reducing cytokine storm as a late complication of critically ill COVID-19. Clin Immunol 2020;214:108408.

33 Ronco C, Reis T. Kidney involvement in COVID-19 and rationale for extracorporeal therapies. Nat Rev Nephrol 2020;16:308-10.

$34 \mathrm{NIH}$. Clinicaltrials.Gov: U.S. National library of medicine, 2020https:// clinicaltrials.gov/ct2/results?cond=COVID-19\&term=Intensive+Care\& cntry $=\&$ state $=\&$ city $=\& d i s t=\&$ Search $=$ Search [Accessed 17 Oct 2020]

35 NSW. Australian New Zealand clinical trials Regsitry. Sydney: NHMRC Clinical Trials Centre, University of Sydney (Australia), 2020.

36 Nasrallah AA, Farran SH, Nasrallah ZA, et al. A large number of COVID-19 interventional clinical trials were registered soon after the pandemic onset: a descriptive analysis. J Clin Epidemiol 2020;125:170-8.

37 Karlsen APH, Wiberg S, Laigaard J, et al. A systematic review of trial registry entries for randomized clinical trials investigating COVID-19 medical prevention and treatment. PLoS One 2020;15:e0237903.

38 Hessey E, Milovanovic L, Sebastianski M, et al. Epidemiology and clinical characteristics of critically ill patients with coronavirus 2019 (COVID-19): a rapid review and meta-analysis. Front Med 2020;7. doi:10.3389/fmed.2020.00295

39 Wang Y, Zhang D, Du G, et al. Remdesivir in adults with severe COVID-19: a randomised, double-blind, placebo-controlled, multicentre trial. Lancet 2020;395:1569-78.

40 Higgins JPT, Altman DG, Gøtzsche PC, et al. The Cochrane collaboration's tool for assessing risk of bias in randomised trials. BMJ 2011;343:d5928.

41 Peterson J, Welch V, Losos M, et al. The Newcastle-Ottawa scale (NOS) for assessing the quality of nonrandomised studies in metaanalyses. Ottawa: Ottawa Hospital Research Institute, 2011.

42 Stroup DF, Berlin JA, Morton SC, et al. Meta-Analysis of observational studies in epidemiology: a proposal for reporting. JAMA 2000;283:2008-12.

43 DerSimonian R, Laird N. Meta-Analysis in clinical trials. Control Clin Trials 1986;7:177-88.

44 Higgins JP, Thomas J, Chandler J, et al. Cochrane Handbook for systematic reviews of interventions. John Wiley \& Sons, 2019

45 Tobaiqy M, Qashqary M, Al-Dahery S, et al. Therapeutic management of COVID-19 patients: a systematic review. Int $J$ Infect Control2020:100061

46 Cao B, Wang Y, Wen D, et al. A trial of Lopinavir-Ritonavir in adults hospitalized with severe Covid-19. N Engl J Med 2020;382:1787-99.

47 Armstrong RA, Kane AD, Cook TM. Outcomes from intensive care in patients with COVID-19: a systematic review and meta-analysis of observational studies. Anaesthesia 2020;75:1340-9.

48 Huang $\mathrm{D}$, Lian X, Song F, et al. Clinical features of severe patients infected with 2019 novel coronavirus: a systematic review and metaanalysis. Annals of translational medicine 2020;8.

49 ClinicalTrials.gov. Efficacy of early administration of tocilizumab in COVID-19 patients Bethesda (MD): National library of medicine (US), 2020. Available: https://clinicaltrials.gov/ct2/show/NCT04346355 [Accessed 29 May 2020].

50 ClinicalTrials.gov. Awake prone positioning to reduce invasive ventilation in COVID-19 induced acute respiratory failurE (APPROVECARE) Bethesda (MD): National library of medicine (US), 2020. Available: https://clinicaltrials.gov/ct2/show/NCT04347941 [Accessed 29 May 2020].

51 Angus DC, Berry S, Lewis RJ, et al. The REMAP-CAP (randomized embedded multifactorial adaptive platform for community-acquired pneumonia) study. rationale and design. Ann Am Thorac Soc 2020;17:879-91.

52 Tan D. COVID-19 Ring-based prevention trial with Lopinavir/Ritonavir CORIPREV-LR, 2020

53 Ins. Solidarity: an international randomized controlled trial to evaluate non-licensed COVID-19 treatments in addition to standard of care among hospitalized patients international clinical trials registry platform (ICTRP2020), 2020. Available: https://www.ins.gob.pe/ ensayosclinicos/rpec/recuperarECPBNuevoEN.asp?numec $=010-20$ [Accessed 8 Jun 2020].

54 Viner RM, Whittaker E. Kawasaki-like disease: emerging complication during the COVID-19 pandemic. Lancet 2020;395:1741-3.

55 Zhang L, Feng X, Zhang D, et al. Deep vein thrombosis in hospitalized patients with COVID-19 in Wuhan, China: prevalence, risk factors, and outcome. Circulation 2020;142.

56 Worldometer. COVID-19 Coronavirus Pandemic Worldometers. info, 2020. Available: https://www.worldometers.info/coronavirus/ [Accessed 19 Oct 2020]. 